\title{
Microbial-based biostimulant enhances sweet pepper performance by metabolic reprogramming of phytohormone profile and secondary plant metabolism
}

\author{
Paolo Bonini ${ }^{1}$, Youssef Rouphael ${ }^{2}$, Maria Begona Miras Moreno $^{3}$, Byungha Lee ${ }^{4}$, \\ Mariateresa Cardarelli ${ }^{5}$, Gorka Erice ${ }^{6}$, ${\text { Veronica } \text { Cirino }^{6} \text {, Luigi Lucini }}^{3}$, and Giuseppe Colla ${ }^{7}$ \\ ${ }^{1}$ Affiliation not available \\ ${ }^{2}$ University of Naples Federico II \\ ${ }^{3}$ Università Cattolica del Sacro Cuore Facoltà di Scienze Agrarie Alimentari e Ambientali \\ ${ }^{4}$ NGAlab \\ ${ }^{5}$ CREA Centro di Ricerca Orticoltura e Florovivaismo \\ ${ }^{6}$ Atens \\ ${ }^{7}$ Universita degli Studi della Tuscia
}

May 20, 2020

\begin{abstract}
Microbial-based biostimulants can improve crop productivity by modulating cell metabolic pathways including hormonal balance. However, little is known about the microbial-mediated molecular changes causing yield increase. The present study elucidates the metabolomic modulation occurring in pepper (Capsicum annuum L.) leaves at the vegetative and reproductive phenological stages in response to microbial-based biostimulants containing the arbuscular mycorrhizal fungi Rhizoglomus irregularis and Funneliformis mosseae as well as Trichoderma koningii. Application of endophytic fungi significantly increased total fruit yield by $23.7 \%$ compared to that of untreated plants. Multivariate statistics indicated that the biostimulant treatment substantially altered the shape of the metabolic profile of pepper. Compared to the untreated control, the plants treated with microbial biostimulants presented with modified gibberellin, auxin, and cytokinin production and distribution. The biostimulant treatment also induced secondary metabolism and caused carotenoids, saponins, and phenolic compounds to accumulate in the plants. Differential metabolomic signatures indicated diverse and concerted biochemical responses in the plants following the colonisation of their roots by beneficial microorganisms. The above findings demonstrated a clear link between microbialmediated yield increase and a strong up-regulation of hormonal and secondary metabolic pathways associated with growth stimulation and crop defence to environmental stresses.
\end{abstract}

\section{Summary statement}

Root inoculation with arbuscular mycorrhizal fungi Rhizoglomus irregularis and Funneliformis mosseae as well asTrichoderma koningii increased fruit yield of greenhouse pepper through a general up-regulation of hormonal and secondary metabolic pathways associated with growth stimulation and crop defence to environmental stresses.

\section{Introduction}

Three major current global challenges are food security, environmental degradation, and climate change. The first may be augmented and the latter two diminished by improving nutrient (nitrogen, phosphorus) use efficiency in agricultural crop production and stabilising yield by practicing sustainable agricul- 
ture (Searchinger, Waite, Hanson, Ranganathan, \& Dumas, 2018). The application of plant biostimulants such as beneficial microbes (arbuscular mycorrhizal fungi [AMF], Trichoderma spp., plant growth-promoting rhizobacteria [PGPR]), and bioactive substances (humic and fulvic acids, macroalgae and microalgae, protein hydrolysates, and silicon) used either separately or in combination may help crops contend with the aforementioned challenges (Rouphael \& Colla, 2020).

Plant biostimulants were recently defined in the Regulations of the European Parliament and Council (Regulation EU 2019/1009) as “... EU fertilising product(s) able to stimulate plant nutrition processes independently of the product's nutrient content with the sole aim of improving one or more of the following characteristics of the plant or the plant rhizosphere: 1) nutrient use efficiency, 2) tolerance to abiotic stress, 3) quality traits, or 4) availability of confined nutrients in the soil or rhizosphere". AMF comprise a very important category of biostimulants (Rouphael et al., 2015). They are members of the Glomeromycotina family and establish mutualistic relationships with $74 \%$ of all terrestrial plant species (Spatafora et al., 2016). AMF boost productivity and enhance tolerance to abiotic stress (high temperature, drought, and salinity) in crops. AMF inoculation enhances the growth and vigour of the root apparatus in terms of biomass, length, density, and branching. It improves macronutrient ( $\mathrm{N}, \mathrm{P}$, and $\mathrm{Fe}$ ) and micronutrient ( $\mathrm{Mn}$ and $\mathrm{Zn})$ uptake and assimilation. It ameliorates water relations and photosynthetic activity, upregulates secondary metabolism, and releases low- and high-molecular-weight organic compounds such as amino acids, phenolics, organic acids, and proteins into the rhizosphere. It also modulates phytohormone signalling (Bernardo et al., 2018; Lucini et al., 2019; Rouphael and Colla, 2018; Rouphael et al., 2015; Yakhin, Lubyanov, Yakhin, \& Brown, 2017). The indirect and direct mechanisms of AMF influence shoot and root function and augment crop agronomic performance. Other plant beneficial endophytic fungi includeTrichoderma spp. Several of them are registered as microbial biological control agents (López-Bucio, Pelagio-Flores, \& Herrera-Estrella, 2015). However, several studies reported that certain Trichoderma spp. including T. atroviride, T. koningii , T. harzianum, and T. virens are also plant biostimulants that boost crop performance and nutrient use efficiency and/or endue plants with abiotic stress tolerance (Colla, Rouphael, Di Mattia, El-Nakhel, \& Cardarelli, 2015; Saia et al., 2019). The direct and indirect mechanisms of the biostimulant action of Trichodermastrains include i) improvement of lateral root development, ii) induction of plant mitogen-activated protein 6 , and iii) production and rhizosphere excretion of auxins and secondary metabolites such as volatile and nonvolatile substances that stimulate various plant responses and enhance crop nutrient uptake, resilience, and productivity (Lopez-Bucio et al., 2015).

The beneficial effects of combinations of AMF and Trichoderma on vegetable crops were previously demonstrated under both optimal and suboptimal conditions (Colla et al., 2015; Saia et al., 2019). However, the physiological and molecular mechanisms underlining biostimulant action have not been fully elucidated. One strategy to clarify biostimulant efficacy is to analyse metabolic profiling. In turn, this process serves as a basis for subsequent transcriptomic analyses. Metabolomic phytochemical characterisation could identify numerous physiological processes and metabolic pathways modulated by biostimulants (Yakhin et al., 2017).

It has been hypothesised that AMF and Trichoderma can induce an enhance fruit yield by modulating the hormonal balance and secondary metabolic pathways.

In the present study, then, an untargeted metabolomics approach was conducted on greenhouse pepper. The objectives were to illuminate metabolomic reprogramming by microbial biostimulants in leaf tissue at the vegetative and reproductive phenological stages, elucidate biostimulant regulation of key phytohormones, and correlate these molecular-level biostimulant-promoted changes to observed fruit yield and quality variations.

\section{Materials and Methods}

Growth conditions, plant material, crop management, and experimental design

The trial was conducted in a greenhouse located at Paraje Aguilas Bajas, Santa Maria del Aguila, Almeria, Spain (36deg47'39"N 2 $46^{\prime} 32^{\prime \prime} \mathrm{W}$ ). The greenhouse was composed of polycarbonate walls and a roof made of trilaminated low-density polyethylene (LDPE) film (200 $\mu$ m thickness) with $\sim 60 \%$ spectral transmittance in the photosynthetically active radiation (PAR) region. The greenhouse was unheated and passively ventilated 
with lateral side panels and flap roof windows. It had an east-west orientation and a north-south crop row alignment. The air temperature and relative humidity $(\mathrm{RH})$ inside the greenhouse were in the ranges of $12-$ $32{ }^{\circ} \mathrm{C}$ and $50-70 \%$, respectively. Transplants of the sweet pepper (Capsicum annuum L.) hybrid 'SV1204PB' (Seminis, Montornés del Vallés, Barcelona, Spain) at the 4-5 true-leaf stage were planted in "Enarenado" sandy soil commonly used in greenhouse production in Almería. The planting date was 19 July 2017 and the planting density was $2.0 \mathrm{~m}^{-2}$. The soil composition was $13.5 \%(\mathrm{w} / \mathrm{w})$ clay, $72.8 \%(\mathrm{w} / \mathrm{w})$ sand, and $13.7 \%(\mathrm{w} / \mathrm{w})$ silt. Its $\mathrm{pH}$ was 7.52 , its organic matter content was $0.71 \%$, and its total nitrogen, available phosphorus, and exchangeable potassium were $690 \mathrm{mg} \mathrm{kg}^{-1}, 51.4 \mathrm{mg} \mathrm{kg}^{-1}$, and $321 \mathrm{mg} \mathrm{kg}^{-1}$, respectively. Aerial drip irrigation was used. The in-line emitters were positioned at $0.30-\mathrm{m}$ intervals and the emitter flow rate was $3.4 \mathrm{~L} \mathrm{~h}^{-1}$. Preplant fertiliser was broadcast at $90 \mathrm{~kg}^{*} \mathrm{ha}^{-1} \mathrm{P}, 120 \mathrm{~kg}^{*} \mathrm{ha}^{-1} \mathrm{~K}$, and $15 \mathrm{~kg}^{*} \mathrm{ha}^{-1} \mathrm{Mg}$ and incorporated into the soil. Additional fertiliser in the form of $\mathrm{K}_{2} \mathrm{SO}_{4}\left(80 \mathrm{~kg}^{*} \mathrm{ha}^{-1} \mathrm{~K}\right)$ was applied through the drip irrigation system. Nitrogen was applied via fertigation in the form of $27 \% \mathrm{NH}_{4} \mathrm{NO}_{3}$ soluble fertiliser starting $10 \mathrm{~d}$ after transplanting until day 83. The total $\mathrm{N}$ supply was split into ten weekly dressings. Powdery mildew caused by Leveillula taurica was controlled by three foliar applications of penconazole (Topas 10EC; Syngenta, Madrid, Spain) at the label-recommended rate. Aphids and spider mites were controlled by one foliar application each of imidacloprid (Confidor 200 SL; Bayer Crop Science, Valencia, Spain) and fenpyroximate (Miro; Bayer Crop Science, Valencia, Spain), respectively. Bumblebees promoted flower pollination. Weeds were controlled by hand hoeing. The control and microbial-based biostimulant treatments were compared in a randomised block design with four replicates for a total of eight experimental plots. The microbial-based biostimulants were applied through a drip irrigation system. The first application was made at $15 \mathrm{~d}$ after transplanting (DAT) (3 August 2017) at the rates of $1 \times 10^{-6}$ spores ha $^{-1}$ Rhizoglomus irregularis BEG72 and 1 $10^{-6}$ spores ha ${ }^{-1}$ Funneliformis mosseae BEG234 in the form of $2.0 \mathrm{~kg} \mathrm{ha}^{-1}$ Team Horticola (Agrotecnologias Naturales, S.L., Tarragona, Spain) plus $1 \times 10^{12} \mathrm{CFU} \mathrm{a}^{-1}$ Trichoderma koningii TK7 in the form of $1.0 \mathrm{~kg} \mathrm{ha}^{-1}$ Condor Shield (Agrotecnologias Naturales, S.L., Tarragona, Spain). The second treatment was applied 43 DAT (31 August 2017) at the rate of $5 \times 10^{11} \mathrm{CFU}^{-1}$ ha Trichoderma koningii TK7 as $0.5 \mathrm{~kg}$ Condor Shield (Agrotecnologias Naturales, S.L., Tarragona, Spain). Each experimental plot was $30 \mathrm{~m}^{2}$ and contained 60 plants.

Yield measurements and arbuscular mycorrhizal fungi (AMF) root colonisation

Fully mature pepper fruits were harvested from 139 DAT (5 December 2017) to 272 DAT (17 April 2018). Mean fruit weight and number and marketable yield were determined for each experimental plot (replicate). Rotten fruit and those weighing $<100 \mathrm{~g}$ were considered unmarketable yield.

At the end of the trial, the roots of six pepper plants per experimental plot were rinsed and subsamples were used to evaluate AMF root colonisation. The root samples were cleared with $10 \%$ (w/v) KOH, stained with $0.05 \%$ (w/v) trypan blue in lactophenol, and microscopically (Stereo microscope Leica EZ4V, 32x Leica Microsystems Srl, Buccinasco, Italy) examined for AMF colonisation. The percentage of colonised root segments was determined by the gridline intercept method (Giovannetti \& Mosse, 1980).

\section{Quantitative PCR determination of Trichodermaconcentration in rhizosphere soil}

At the end of the trial, rhizosphere soil was collected by shaking the roots collected from ten plants per plot. To determine the Trichoderma concentration in the rhizosphere, DNA was extracted from the soil samples with a DNeasy ${ }^{(\mathrm{r})}$ Powersoil $^{(\mathrm{r})}$ kit (Cat. No. 12888-50; Qiagen, Hilden, Germany) according to Qiacube (Qiagen, Hilden, Germany) automation procedures. To amplify the transcripts, the genus-specific primer pair RM3/RM4 was used to detect Trichoderma spp. Every 4- $\mu \mathrm{L}$ DNA sample was amplified in a 20- $\mu \mathrm{L}$ reaction system in the presence of $10 \mu \mathrm{L}$ QuaniNova ${ }^{\mathrm{TM}} \mathrm{SYBR}^{\circledR}$ Green Supermix $(2 \mathrm{x})$ and $0.14 \mu \mathrm{L}$ of $25 \mu \mathrm{M}$ primers on a Rotor-Gene Q (Qiagen, Hilden, Germany). The qPCR cycling conditions were as follows: initial incubation at $95{ }^{\circ} \mathrm{C}$ for $2 \mathrm{~min}, 45$ cycles of $95{ }^{\circ} \mathrm{C}$ for $5 \mathrm{~s}$ each, and $60{ }^{\circ} \mathrm{C}$ for $12 \mathrm{~s}$. Two technical replicates were performed per sample. After qPCR, the absolute values for the transcript quantities were calculated by interpolation of standard curves plotted using serial DNA dilutions, namely, undiluted and 1:1,000, 1:10,000, and 1:100,000 dilutions of standardised liquid culture at a total Trichoderma spp. concentration of $10^{9}$ (CFU) $\mathrm{mL}^{-1}$. 
Sample collection and untargeted metabolomics

Four leaves in the third position from the branch tip were harvested for untargeted metabolomics at 43 DAT (31 August 2017) and at 131 DAT (27 November 2017). The leaves were flash-frozen in liquid nitrogen and stored at $-80{ }^{\circ} \mathrm{C}$ until subsequent metabolomic analysis.

An untargeted metabolomics approach was conducted in the UHPLC 1290 chromatographic system coupled to a hybrid quadrupole-time-of-flight (Q-TOF) G6550 mass spectrometer (UHPLC/Q-TOF) (Agilent Technologies, Santa Clara, CA, USA). A Waters Acquity UPLC ${ }^{\circledR}$ BEH C18 column $(100 \times 2.1$ mm i.d., $1.7 \mu \mathrm{m}$ ) (Waters Corp., Milford, MA, USA) was used for reverse-phase chromatographic separation. The binary gradient consisted of water and acetonitrile and the Riken Plasma method was followed (Tsugawa et al., 2019). The injection volume was $2 \mu \mathrm{L}$ and the mass spectrometer was run in positive polarity and SCAN mode (range: 100-1,700 m/z; extended dynamic range setting). Quality controls (QC) were prepared by pooling $10-\mu \mathrm{L}$ samples. Five QCs were acquired in data-dependent mode (auto MS/MS) at $1 \mathrm{~Hz}, 10$ precursors/cycle, collision energies of $10 \mathrm{~V}, 30 \mathrm{~V}$, and $50 \mathrm{~V}$ ), and in iterative mode with active exclusion to increase the number of compounds targeted for tandem MS fragmentation.

Alignment, blank filtration, and identification were performed in MSDIAL v. 4.0 (Riken, Tokyo, Japan) using the publicly available library MoNA (Mass Bank of North America) and an internal standard compound library as specified in the Supplementary Table 1. Compounds lacking experimental MS/MS spectra were annotated with MSFINDER (Riken, Tokyo, Japan) following the procedure described in Blazenovic et al. (2019). The alternatives were filtered by retention time prediction (Bonini et al., 2020). MSI (metabolomics standards initiative) levels for each identified compound are listed in Supplementary Table 1.

Statistics and data analysis

Data were statistically analysed with SPSS v. 21 (IBM Corp., Armonk, NY, USA). The microbial-based biostimulant effects were analysed by an unpaired Student's $t$-test. The compound intensity table exported from MSDIAL v. 4.0 (Riken, Tokyo, Japan) (Tsugawa et al., 2015) was uploaded into MS-FLO (Riken, Tokyo, Japan) (DeFelice et al., 2017) to reduce false positives and duplicates. The output was imported into R v. 3.6.0 for centring, scaling, PCA, and calculation of fold changes, Benjamini-Hochberg false discovery rates (FDR), and corrected p-values. Venn diagrams were plotted to identify metabolites common to two sampling points but not exclusive to a particular growth stage. Compounds with $\mathrm{P}<0.05$ were imported into ChemRICH (Barupal \& Fiehn, 2017) for enrichment analysis based on their chemical similarity and MetaMapp (Barupal et al., 2012) for chemical network analysis. Cytoscape (Saito et al., 2012) displayed exported MetaMapp data and plotted the final images.

\section{Results}

Soil fungal concentration and crop yield

By the end of the trial, the percentage of mycorrhizal root colonisation was significantly $(P<0.01)$ higher under the microbial inoculation treatment (33.6\%) than it was under the uninoculated control treatment (8.0\%). The total number of Trichodermacolonies recovered from the rhizosphere under the microbial-based treatment was significantly $(P<0.01)$ higher than that recorded for the untreated control $\left(2.2 \times 10^{5} \mathrm{vs} .1 .2\right.$ $\times 10^{3} \mathrm{CFU} \mathrm{g}^{-1}$, respectively).

Relative to the uninoculated control, inoculation with AMF and Trichoderma koningii significantly increased fruit yield especially during the first part of the reproductive cycle, namely, early yield 139 DAT (P<0.05) and 174 DAT $(\mathrm{P}<0.01)$ (Table 1$)$. The comparatively higher production rates measured at 139 DAT and 272 DAT for pepper plants inoculated with microbial-based biostimulant was due to an increase in mean fruit weight. In contrast, the relatively higher fruit yield determined for 174 DAT was attributed to increases in both fruit number per plant and mean fruit mass (Tables 1-3). The microbial-based biostimulant significantly improved cumulative fruit yield by $23.7 \%$ relative to uninoculated pepper plants (Table 1 ).

Modulation of metabolomic profile 
Here, ultra-high-performance liquid chromatography quadrupole-time-of-flight high-resolution mass spectrometry (UHPLC-QTOF) and annotation in publicly available databases and large metabolite groups were conducted to obtain wide metabolome coverage. We applied UHPLC-QTOF-based untargeted metabolomic profiling of crude extracts to assess relative differences in the vegetative stage (43 DAT) and reproductive stage (131 DAT) leaf metabolite profiles between inoculated and uninoculated plants. A principal component analysis (PCA) explained $79 \%$ of the overall variance. The PCA score plot (Figure 1) showed two main clusters accounting for the vegetative and reproductive stages, respectively. Within each cluster, good separation was achieved between the metabolomic profiles of leaves from inoculated plants and those of leaves harvested from the uninoculated control. This finding was anticipated as the metabolism dramatically shifts as the plant transitions from the vegetative to reproductive phases. This outcome was relevant as PCA provides unsupervised descriptions of relatedness/unrelatedness. We ran a $t$-test ANOVA $(\mathrm{P}<0.01)$ to identify differentially accumulated metabolites at each plant growth stage. This analysis disclosed $>466$ annotated metabolites (Supplementary Tables 2 and 3) that had significantly changed between the vegetative and reproductive stages. Of these, 327 were common to both the first and second sampling points (Figure 2). In contrast, 68 and 71 metabolites differentially accumulated during the vegetative and reproductive stages, respectively (Figure 2). The interactions between microbial inoculants and plants are complex. Nevertheless, metabolomics effectively included the metabolic responses and mechanisms involved in the plant-microbe interactions. As $70 \%$ of the metabolites were common to the vegetative and reproductive phenological stages (Figure 2), biostimulant-mediated shifts in the leaf metabolome were recorded for both of them. On the other hand, certain stage-specific responses could be identified as well.

To clarify and visualise the variations between metabolic profiles at the vegetative and reproductive stages, we performed a chemical enrichment analysis using ChemRICH (Figure 3; Tables 4 and 5) and plotted the output by MetaMapp Cytoscape (Figure 4) (Barupal \& Fiehn, 2017). Most of the significantly upregulated and downregulated metabolites (fold-change values [?] 0.5 and [?] 1.5, respectively; P [?] 0.01) had a wide range of functions including growth stimulation, antifungal activity, pathogen resistance, energy sources, and secondary signalling cofactors.

\section{Discussion}

There is a growing interest in the use of beneficial microbial inoculants such as AMF, Trichoderma spp., and PGPR in horticulture as they have multiple beneficial effects on crops (Lopez-Bucio et al., 2015; Rouphael et al., 2015). In the present study, we inoculated pepper plants with the AMF species Rhizoglomus irregularis and Funneliformis mosseae. Trichoderma koningiienhanced mycorrhizal root colonisation whilst Trichodermarhizosphere population accelerated and increased total crop yield by $24 \%$ relative to uninoculated plants (Table 1). The increase in pepper yield was attributed to gains in fruit weight and/or number. Colla et al. (2014) reported that compared with uninoculated field-grown zucchini plants, those supplied with live AMF G. intraradices and T. atroviride inocula presented with greater early and total yields. The beneficial fungi act as phytostimulants and improve foliar nutrient content. The phytostimulatory efficacy of beneficial fungi is explained by complex signal exchange and crosstalk between the host plants and the microorganisms affecting phytohormone balance and plant metabolism (Sbrana, Turrini, \& Giovannetti, 2017). Metabolomics helps elucidate the metabolic pathways and processes involved in plant-microbe interactions. Growth stage has a hierarchically strong effect on the leaf metabolome. Nevertheless, microbial biostimulants significantly alter the metabolome such that it is readily distinguishable from the control (Figure 1).

The microbial treatments elicited several processes related to plant secondary metabolism. Microbial-based biostimulants promote the accumulation of carotenoids and other terpenes, saponins, phenolic compounds, and phospholipids (Figures 3 and 4).

Plant responses to microbial-based biostimulants involve modulations of the phytohormone network. Treatments with beneficial fungi alter auxins, cytokinins, and gibberellins. Modification of the hormone profile may have been associated with the observed yield increases. Several studies demonstrated that microbial biostimulants promote yield by changing the phytohormone balance, increasing nutrient availability and uptake, and enhancing abiotic stress tolerance (Rouphael et al., 2015; Saia et al., 2019, 2020). Certain putative 
mechanisms for the biostimulant activity of microbial-based inoculant (AMF + Trichoderma) in pepper have been proposed. Microbial-based inoculant augments root biomass, length, density, and branching which, in turn, increases macronutrient and micronutrient uptake and boosts crop productivity. It also regulates key phytohormones such as gibberellins, cytokinins, and auxins (Lopez-Bucio et al., 2015; Lucini et al., 2019; Rouphael et al., 2015).

Gibberellins are diterpenoid phytohormones that regulate plant development, flowering, and senescence (Shu, Zhou, Chen, Luo, \& Yang, 2018). In response to microbial-based inoculant treatment, gibberellins A81, A36, A37, A12, and A20 increased by 1.3-16x relative to the control at both sampling points. Coordination between gibberellin biosynthesis and oxidation affects pollination and fruit set in tomato (Serrani, Sanjuan, Ruiz-Rivero, Fos, \& Garcia-Martinez, 2007). Gibberellin A20 was recently linked to increased maize yield (Tucker et al., 2019).

Here, the microbial-based biostimulant treatment induced the auxins indole-3-acetamide and indole-3-pyruvic acid by 1.7-7.5x relative to the control. Auxins upregulate the genes encoding oxidases regulating gibberellin metabolism (Frigerio et al., 2006). Auxins and gibberellins overlap in terms of root growth and fruit set regulation (Bermejo et al., 2018). Microbial-based biostimulant also increased the accumulation of the cytokinin trans -zeatin by 2.2-5.1x in pepper leaves compared with the control. Cytokinins interact with auxins to fine-tune root and shoot development. Trans -zeatin modulates meristem activity and mediates plant responses to variable extrinsic factors such as abiotic stress (Werner \& Schmulling, 2009). We postulate that coordinated auxin, gibberellin, and cytokinin recruitment may have contributed to the enhanced pepper fruit yield observed here. Earlier studies reported that beneficial microbes promote plant growth and productivity by altering phytohormone status (Bhattacharyya \& Jha, 2012).

Modulation of plant signalling compounds in response to the microbial-based biostimulant treatment also involved membrane lipids. Phospholipids are plasma membrane components that play important roles in cell signalling, membrane trafficking, and apoptosis (Xue, Chen, \& Mei, 2009). The microbial-based biostimulant treatment changed the phospholipids profile. It altered twenty foliar metabolites at the first sampling (vegetative stage) and 31 foliar metabolites in the second sampling (reproductive stage). Accumulation of phosphatidylethanolamines (PE(P-16:0/20:5)), phosphatidic acid (PA(15:0/22:6), PA(O-18:020:3)), phosphatidylinositol (PIM4(18:1/14:0)), and phosphatidylserine (PS(P-16:013:0)) increased by 1.5-30x in the biostimulant-treated plants compared to the control. Relative to the untreated leaves, lysophospholipids (PA(P-16:0e18:2)) increased by 6.5x in biostimulant-treated leaves from the second sampling (reproductive stage). Lysophospholipids release calcium from the endoplasmic reticulum, promote cell division, and inhibit apoptosis (Hou, Ufer, \& Bartels, 2016; Ye, 2008).

The microbial treatment also modulated the biosynthesis of carotenoids, saponins, phenolic compounds, and purines. Secondary metabolism is often altered in response to plant interactions with the ambient environment including agronomic practices and plant-microbe interactions (Yang et al., 2018). Here, metabolomics identified substantial alterations in secondary metabolism. Hence, plant responses to microbial biostimulants entails the coordinated modulation of several unrelated pathways.

Compared to the control, by the second sampling date, foliar vitamin $\mathrm{A}$ and $\alpha$-carotene were $1.5 \times$ and $8.5 \times$ higher, respectively, following microbial biostimulant treatment. Carotenoids absorb light energy, participate in photosynthesis, protect plants against oxidative damage, and are precursors of visual pigment chromophores and volatile apocarotenoids that attract pollinators (Heath, Cipollini, \& Stireman, 2013; Sun, et al., 2018). Moreover, they are involved in plant responses to abiotic stresses and plant-microbe interactions (Felemban, Braguy, Zurbriggen, \& Al-Babili, 2019). Blumenols comprise a class of apocarotenoids or cyclohexanone derivatives of carotenoid cleavage. They also accumulated in the biostimulant-treated plants. Blumenols accumulate in the roots and shoots of mycorrhized plants and have been proposed as arbuscular mycorrhizal fungi colonisation markers (Wang et al., 2018). Relative to the control, blumenol B was $2 \times$ and $2.5 \times$ higher at the first and second sampling dates, respectively, after biostimulant application. Their functions in processes other than allelopathy are unknown. However, their levels are strongly correlated with the degree of mycorrhization (Fester et al., 2002). Here, foliar saponins were $1.5-10 \times$ higher in plants treated 
with biostimulant than the untreated control. Saponins are produced constitutively in plants and comprise part of plant defence. They have both antifungal and antifeedant activity. Though they are generally associated with pathogenesis, it was recently reported that saponins may participate in mutualistic relationships among plants, rhizobacteria, and mycorrhizae (Szakiel, Paczkowski, \& Henry, 2011).

Compared with the control, plants subjected to the microbial treatments presented with higher levels of phenolic compounds. Phenolic metabolites are essential for lignin and pigment biosynthesis and participate in plant responses to pathogens and external stimuli (Bhattacharya, Sood, \& Citovsky, 2010). Mycorrhizae elicit phenolic biosynthesis in other plant species (Baslam \& Goicoechea, 2012; Jugran et al., 2015). They also trigger plant defence against abiotic and biotic stresses and improve nutrient availability and use efficiency (Bernardo et al., 2019; Sharma \& Sharma, 2017). Here, irrespective of growth stage, skullcapflavone I, pelargonidin-3-o-glucoside, kaempferol, genistein, apiin, and myricatomentoside I accumulated to levels 3$87 \times$ higher in the biostimulant-treated plants than the control. Phenolics are associated with plant defence mechanisms. Flavones may protect plants from both biotic and abiotic stress (Martinez et al., 2016). Lignans have high antioxidant activity (Durazzo, Turfani, Azzini, Maiani, \& Carcea, 2013; Hu, Yuan, \& Kitts, 2007). Compared with the uninoculated, the gibbilimbol B level was $1.5 \times$ and $4.2 \times$ higher at the first and second sampling dates, respectively, in the inoculated plants. Gibbilimbol B was reported to have fungicidal activity against Fusarium oxysporum f. sp. dianthi . Coumarin upregulation is related to iron nutrition (Curie \& Mari, 2017), allelochemistry (Niro et al., 2016), and abiotic stress tolerance (Saleh \& Madany, 2015) in plants. Plant coumarins may influence the shape of the root microbiome (Voges, Bai, Schulze-Lefert, \& Sattely, 2019).

Relative to the control, the levels of several purines were altered in the plants treated with the microbial biostimulant here. Several studies have focused on the effects of increased levels of adenosine and purines. These compounds are recycled by the so-called "salvage pathway" (Ashihara, Stasolla, Fujimura, \& Crozier, 2018). At the first and second samplings, we observed sharp increases in the guanosine $(2.7 \times$ and $8.7 \times$, respectively) and N6-threonylcarbamoyladenosine $(3 \times$ and $7.8 \times$, respectively) levels after microbial biostimulant inoculation. Nicotinamide adenine dinucleotide (NAD) and flavin adenine dinucleotide (FAD) are reducing equivalent exchange cofactors that participate in several redox reactions. At both the first and second samplings, NAD and FAD had increased by $1.5-4.4 \times$ in the biostimulant-treated plants relative to the control.

Our metabolomics study revealed that microbial biostimulant treatment had two major effects on pepper. First, the biostimulant modulated the phytohormone profile and phospholipid signalling in the plants. Next, it altered various secondary metabolic processes involving saponins, blumenols, carotenoids, and phenolic compounds. Phytohormones and biochemical messengers are associated with various metabolic processes (Ashihara et al., 2018) and might account for the observed biostimulant-mediated increases in crop productivity. The secondary metabolites modulated by biostimulant treatment have numerous positive influences on plant productivity such as the enhancement of nutrient uptake and assimilation and biotic and abiotic stress tolerance. The elicitation of secondary metabolism by plant beneficial microbes merits further investigation in terms of abiotic stress tolerance and induced systemic response (ISR) induction. Carotenoids and phenolics improve quality and promote health in many fruits including pepper. Thus, the microbial biostimulant treatments applied here could have nutritional implications as well.

\section{Conclusions}

Recent scientific investigations have focused on improving sustainable farming practices that stabilise yield under optimal and suboptimal conditions and comply with changing legislation regarding the application of low-input agrochemicals. Microbial-based biostimulants (AMF and/or Trichoderma) may sustainably enhance crop productivity. Our greenhouse experiment on pepper confirmed that inoculation with a combination of AMF and Trichoderma increased fruit yield by $23.7 \%$ relative to that of the untreated control. A metabolomics analysis revealed that the biostimulant treatment reprogrammed the plant metabolome. Hence, several biochemical processes underly the observed increase in fruit yield. Here, we disclosed that the biostimulant modulated the phytohormone profile and induced secondary metabolism. Specifically, the 
microbial-based biostimulant upregulated compounds such as carotenoids, saponins, and phenolics that participate in plant nutrition, defence and stress response. Thus, the results of the present study confirm that biostimulant amendments favour stable increases in fruit yield and lead the way towards future investigations into their effects on plants under challenging conditions such as abiotic and biotic stress, environmental perturbations, and physicochemical imbalances.

\section{Data Availability}

Raw metabolomics data are available at the GNPS MASSIVE server (https://massive.ucsd.edu/ProteoSAFe/) under deposit No. MSV000085204.

\section{Acknowledgements}

The authors thank Tobias Kind from UC Davis, Davis, CA, USA for his assistance with manuscript revision.

\section{Conflict of Interest}

Veronica Cirino and Gorka Erice are former employees of Atens Agrotecnologias Naturales.

\section{Author Contributions}

P.B., G.C., Y.R., V.C., M.C. and L.L. designed the experiment. G.C., M.C., Y.R. and G.E. measured and made the interpretation of agronomical data. P.B. and B.L. acquired the metabolomics and qPCR data. P.B., B.M.M. and L.L. analysed the metabolomics data. All authors discussed the results and contributed to the final manuscript.

\section{References}

Ashihara, H., Stasolla, C., Fujimura, T., \& Crozier, A. (2018) Purine salvage in plants. Phytochemistry, 147, 89-124. https://doi.org/10.1016/j.phytochem.2017.12.008

Barupal, D. K.\& Fiehn, O. (2017). Chemical similarity enrichment analysis (ChemRICH) as alternative to biochemical pathway mapping for metabolomic datasets. Scientific Reports , 7(1), 1-11. https://doi.org/10.1038/s41598-017-15231-w

Barupal, D. K., Haldiya, P. K., Wohlgemuth, G., Kind, T., Kothari, S. L., Pinkerton, K. E., \& Fiehn, O. (2012). MetaMapp: mapping and visualizing metabolomic data by integrating information from biochemical pathways and chemical and mass spectral similarity. BMC Bioinformatics, 13(1), 99. https://doi.org/10.1186/1471-2105-13-99

Baslam, M., \& Goicoechea, N. (2012). Water deficit improved the capacity of arbuscular mycorrhizal fungi (AMF) for inducing the accumulation of antioxidant compounds in lettuce leaves.Mycorrhiza , 22 (5), 347359. https://doi.org/10.1007/s00572-011-0408-9

Bermejo, A., Granero, B., Mesejo, C., Reig, C., Tejedo, V., Agustí, M., .. Iglesias, D.J. (2018). Auxin and gibberellin interact in citrus fruit set. Journal of Plant Growth Regulation , 37(2), 491-501. https://doi.org/10.1007/s00344-017-9748-9

Bernardo, L., Carletti, P., Badeck, F. W., Rizza, F., Morcia, C., Ghizzoni, R., .. Lucini, L. (2019). Metabolomic responses triggered by arbuscular mycorrhiza enhance tolerance to water stress in wheat cultivars. Plant Physiology and Biochemistry , 137, 203-212. https://doi.org/10.1016/j.plaphy.2019.02.007

Bhattacharya, A., Sood, P., \& Citovsky, V. (2010). The roles of plant phenolics in defence and communication during Agrobacterium and Rhizobium infection. Molecular Plant Pathology, 11, 705-19. https://doi.org/10.1111/j.1364-3703.2010.00625.x

Bhattacharyya, P. N., \& Jha, D. K. (2012). Plant growth-promoting rhizobacteria (PGPR): emergence in agriculture. World Journal of Microbiology and Biotechnology , 28 (4), 1327-1350. 
Blazenovic, I., Kind, T., Sa, M. R., Ji, J., Vaniya, A., Wancewicz, B., .. Showalter, M. R., 2019. Structure annotation of all mass spectra in untargeted metabolomics. Analytical Chemistry, 91(3), 2155-2162.

Bonini, P., Kind, T., Tsugawa, H., Barupal, D.K., \& Fiehn, O. Retip: retention time prediction for compound annotation in untargeted metabolomics Analytical Chemistry, Just Accepted Manuscript. https://doi.org/10.1021/acs.analchem.9b05765

Colla, G., Rouphael, Y., Canaguier, R., Svecova, E., \& Cardarelli, M. (2014). Biostimulant action of a plant-derived protein hydrolysate produced through enzymatic hydrolysis. Frontiers in Plant Science , 5. https://doi.org/10.3389/fpls.2014.00448

Colla, G., Rouphael, Y., Di Mattia, E., El-Nakhel, C., \& Cardarelli, M. (2015). Co-inoculation of Glomus intraradices andTrichoderma atroviride acts as a biostimulant to promote growth, yield and nutrient uptake of vegetable crops. Journal of the Science of Food and Agriculture, 95(8), 1706-1715. https://doi.org/10.1002/jsfa.6875

Curie, C., \& Mari, S. (2017). New routes for plant iron mining.New Phytologist , 214 (2), 521-525. https://doi.org/10.1111/nph.14364

De Felice, B. C., Mehta, S. S., Samra, S., Cajka, T., Wancewicz, B., Fahrmann, J. F., \& Fiehn, O. (2017). Mass spectral feature list optimizer (MS-FLO): a tool to minimize false positive peak reports in untargeted liquid chromatography-mass spectroscopy (LC-MS) data processing. Analytical Chemistry, 89(6), 32503255. https://doi.org/10.1021/acs.analchem.6b04372

Durazzo, A., Turfani, V., Azzini, E., Maiani, G., \& Carcea, M. (2013). Phenols, lignans and antioxidant properties of legume and sweet chestnut flours. Food Chemistry , 140(4), 666-671. https://doi.org/10.1016/j.foodchem.2012.09.062

Felemban, A., Braguy, J., Zurbriggen, M. D., \& Al-Babili, S. (2019) Apocarotenoids involved in plant development and stress response.Front. Plant Sci. 10, 1168. https://doi.org/10.3389/fpls.2019.01168

Fester, T., Hause, B., Schmidt, D., Halfmann, K., Schmidt, J., Wray, V., .. Strack, D. (2002). Occurrence and localization of apocarotenoids in arbuscular mycorrhizal plant roots. Plant and Cell Physiology , 43 (3), 256-265. https://doi.org/10.1093/pcp/pcf029

Frigerio, M., Alabadí, D., Pérez-Gómez, J., García-Cárcel, L., Phillips, A. L., Hedden, P., \& Blázquez, M. A. (2006). Transcriptional regulation of gibberellin metabolism genes by auxin signaling inArabidopsis. Plant Physiology , 142 (2), 553-563. https://doi.org/10.1104/pp.106.084871

Giovannetti, M., \& Mosse, B. (1980). An evaluation of techniques for measuring vesicular arbuscular mycorrhizal infection in roots. New Phytologist, 84(3), 489-500. https://doi.org/10.1111/j.1469-8137.1980.tb04556.x

Heath, J., Cipollini, D., \& Stireman, J. (2013). The role of carotenoids and their derivatives in mediating interactions between insects and their environment. Arthropod-Plant Interactions,7(1), 1-20. https://doi.org/10.1007/s11829-012-9239-7

Hou, Q., Ufer, G., \& Bartels, D. (2016). Lipid signalling in plant responses to abiotic stress. Plant, Cell and Environment , 39(5), 1029-1048. https://doi.org/10.1111/pce.12666

Hu, C., Yuan, Y. V., \& Kitts, D. D. (2007). Antioxidant activities of the flaxseed lignan secoisolariciresinol diglucoside, its aglycone secoisolariciresinol and the mammalian lignans enterodiol and enterolactone in vitro. Food and Chemical Toxicology, 45(11), 2219-27. https://doi.org/10.1016/j.fct.2007.05.017

Jugran, A. K., Bahukhandi, A., Dhyani, P., Bhatt, I. D., Rawal, R. S., Nandi, S. K., \& Palni, L. M. S. (2015). The effect of inoculation with mycorrhiza: AM on growth, phenolics, tannins, phenolic composition and antioxidant activity in Valeriana Jatamansi Jones. Journal of Soil Science and Plant Nutrition . https://doi.org/10.4067/S0718-95162015005000072 
López-Bucio, J., Pelagio-Flores, R., \& Herrera-Estrella, A. (2015).Trichoderma as biostimulant: exploiting the multilevel properties of a plant beneficial fungus. Scientia Horticulturae, 196 , 109-123. https://doi.org/10.1016/j.scienta.2015.08.043

Lucini, L., Colla, G., Miras Moreno, M. B., Bernardo, L., Cardarelli, M., Terzi, V., .. Rouphael, Y. (2019). Inoculation ofRhizoglomusir regulare or Trichoderma atroviridedifferentially modulates metabolite profiling of wheat root exudates.Phytochemistry 157 , 158-167. https://doi.org/10.1016/j.phytochem.2018.10.033

Martinez, V., Mestre, T. C., Rubio, F., Girones-Vilaplana, A., Moreno, D. A., Mittler, R., \& Rivero, R. M. (2016). Accumulation of flavonols over hydroxycinnamic acids favors oxidative damage protection under abiotic stress. Front. Plant Sci., 7, 838. https://doi.org/10.3389/fpls.2016.00838

Niro, E., Marzaioli, R., De Crescenzo, S., D’Abrosca, B., Castaldi, S., Esposito, A., .. Rutigliano, F. A. (2016). Effects of the allelochemical coumarin on plants and soil microbial community. Soil Biology and Biochemistry , 95, 30-39. https://doi.org/10.1016/j.soilbio.2015.11.028

Rouphael, Y., \& Colla, G. (2020). Editorial: Biostimulants in agriculture. Frontiers in Plant Science, 11, 40. https://doi.org/10.3389/fpls.2020.00040

Rouphael, Y., Franken, P., Schneider, C., Schwarz, D., Giovannetti, M., Agnolucci, M., .. Colla, G. (2015). Arbuscular mycorrhizal fungi act as biostimulants in horticultural crops. Scientia Horticulturae , 196, 91-108. https://doi.org/10.1016/j.scienta.2015.09.002

Rouphael, Y., Lucini, L., Miras-Moreno, B., Colla, G., Bonini, P., \& Cardarelli, M. (2020). Metabolomic responses of maize shoots and roots elicited by combinatorial seed treatments with microbial and nonmicrobial biostimulants. Frontiers in Microbiology 11, 664. https://doi.org/10.3389/fmicb.2020.00664.

Saia, S., Aissa, E., Luziatelli, F., Ruzzi, M., Colla, G., Fica, A. G., .. Rouphael, Y. (2020). Growthpromoting bacteria and arbuscular mycorrhizal fungi differentially benefit tomato and corn depending upon the supplied form of phosphorus. Mycorrhiza , 30 (1), 133-147. https://doi.org/10.1007/s00572-019-00927-w

Saia, S., Colla, G., Raimondi, G., Di Stasio, E., Cardarelli, M., Bonini, P., .. Rouphael, Y. (2019). An endophytic fungi-based biostimulant modulated lettuce yield, physiological and functional quality responses to both moderate and severe water limitation.Scientia Horticulturae , 256, 108595. https://doi.org/10.1016/j.scienta.2019.108595

Saito, R., Smoot, M. E., Ono, K., Ruscheinski, J., Wang, P. L., Lotia, S., ... Ideker, T. (2012). A travel guide to Cytoscape plugins. Nature Methods, 9(11), 1069-1076. https://doi.org/10.1038/nmeth.2212

Saleh, A. M., \& Madany, M. M. Y. (2015). Coumarin pretreatment alleviates salinity stress in wheat seedlings. Plant Physiology and Biochemistry , 88, 27-35. https://doi.org/10.1016/j.plaphy.2015.01.005

Sbrana, C., Turrini, A., Giovannetti, M. (2017). The Crosstalk between plants and their arbuscular mycorrhizal symbionts: a mycocentric view: sign-mediated interactions between cells and organisms. In Biocommunication Sign-Mediated Interactions between Cells and Organisms (eds. R. Gordon \& J. Seckbach), pp. 285-308. World Scientific Publishing Co Pte Ltd, Singapore. https://doi.org/10.1142/9781786340450_0011

Searchinger, T., Waite, R., Hanson, C., Ranganathan, J., \& Dumas, P. (2018). World resources report: creating a sustainable food future. Ed. E. Matthews, pp. 1-97.

Serrani, J. C., Sanjuán, R., Ruiz-Rivero, O., Fos, M., \& García-Martínez, J. L. (2007). Gibberellin regulation of fruit set and growth in tomato. Plant Physiology , 145(1), 246-257. https://doi.org/10.1104/pp.107.098335

Sharma, P. I., \& Sharma, A. K. (2017). Co-inoculation of tomato with an arbuscular mycorrhizal fungus improves plant immunity and reduces root-knot nematode infection. Rhizosphere , 4, 25-28. https://doi.org/10.1016/j.rhisph.2017.05.008 
Shu, K., Zhou, W., Chen, F., Luo, X., \& Yang, W. (2018) Abscisic acid and gibberellins antagonistically mediate plant development and abiotic stress responses. Frontiers in Plant Sciience, 9, 416. https://doi.org/10.3389/fpls.2018.00416

Spatafora, J. W., Chang, Y., Benny, G. L., Lazarus, K., Smith, M. E., Berbee, M. L., .. Gryganskyi A. (2016). A phylum-level phylogenetic classification of zygomycete fungi based on genome-scale data. Mycologia , 108(5), 1028-1046. https://doi.org/10.3852/16-042

Sun, T., Yuan, H., Cao, H., Yazdani, M., Tadmor, Y., \& Li, L. (2018) Carotenoid metabolism in plants: the role of plastids. Molecular Plant 11(1), 58-74. https://doi.org/10.1016/j.molp.2017.09.010

Szakiel, A., Paczkowski, C., \& Henry, M. (2011). Influence of environmental biotic factors on the content of saponins in plants.Phytochemistry Reviews , 10(4), 493-502. https://doi.org/10.1007/s11101-010-9164-2

Tsugawa, H., Cajka, T., Kind, T., Ma, Y., Higgins, B., Iked, K., .. . Arita, M. (2015). MS-DIAL: dataindependent MS/MS deconvolution for comprehensive metabolome analysis. Nature Methods , 12(6), 523526. https://doi.org/10.1038/nmeth.3393

Tsugawa, H., Nakabayashi, R., Mori, T., Yamada, Y., Takahashi, M., Rai, A., ... Kooke, R., (2019). A cheminformatics approach to characterize metabolomes in stable-isotope-labeled organisms. Nature methods , 16(5), 446. https://doi.org/10.1038/s41592-019-0423-x

Tucker, S. L., Dohleman, F. G., Grapov, D., Flagel, L., Yang, S., Wegener, K. M., . . . Bedair, M. (2019). Evaluating maize phenotypic variance, heritability, and yield relationships at multiple biological scales across agronomically relevant environments. Plant, cell \& environment. https://doi.org/10.1111/pce.13681

Voges, M. J. E. E. E., Bai, Y., Schulze-Lefert, P., \& Sattely, E. S. (2019). Plant-derived coumarins shape the composition of anArabidopsis synthetic root microbiome. Proceedings of the National Academy of Sciences of the United States of America , 116(25), 12558 -12565. https://doi.org/10.1073/pnas.1820691116

Wang, M., Schäfer, M., Li, D., Halitschke, R., Dong, C., McGale, E., .. \& Heiling, S. (2018). Blumenols as shoot markers of root symbiosis with arbuscular mycorrhizal fungi. Elife, 7, e37093. https://doi.org/10.7554/elife.37093

Werner, T., \& Schmülling, T. (2009). Cytokinin action in plant development. Current Opinion in Plant Biology , 12(5), 527-538. https://doi.org/10.1016/j.pbi.2009.07.002

Xue, H. W., Chen, X., \& Mei, Y. (2009) Function and regulation of phospholipid signalling in plants. Biochemical Journal , 421(2), 145-156. https://doi.org/10.1042/bj20090300

Yakhin, O. I., Lubyanov, A. A., Yakhin, I. A., \& Brown, P. H. (2017). Biostimulants in plant science: a global perspective. Frontiers in Plant Science, 7, 2049. https://doi.org/10.3389/fpls.2016.02049

Yang, L., Wen, K. S., Ruan, X., Zhao, Y. X., Wei, F., \& Wang, Q. (2018). Response of plant secondary metabolites to environmental factors. Molecules, 23(4), 762. https://doi.org/10.3390/molecules23040762

Ye, X. (2008) Lysophospholipid signaling in the function and pathology of the reproductive system. Human reproduction, 14(5), 519-536. https://doi.org/10.1093/humupd/dmn023

\section{FIGURES:}




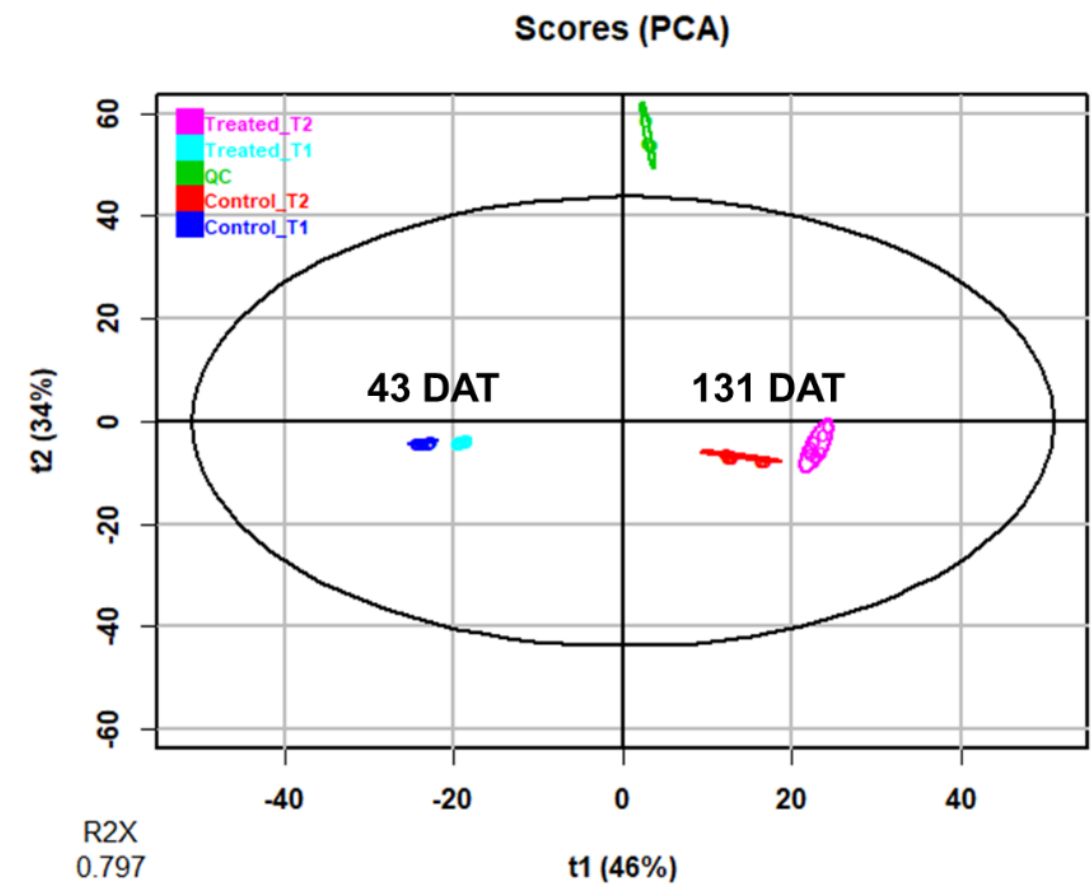

Figure 1. Principal Component Analysis $(\mathrm{PCA})$ of statistically different metabolites $(\mathrm{P}<0.05)$ in pepper plants following treatment with microbial biostimulants. Compounds were profiled by untargeted metabolomics and samples harvested at two dates: 43 days after transplanting (DAT; vegetative stage), and 131 DAT (reproductive stage). 


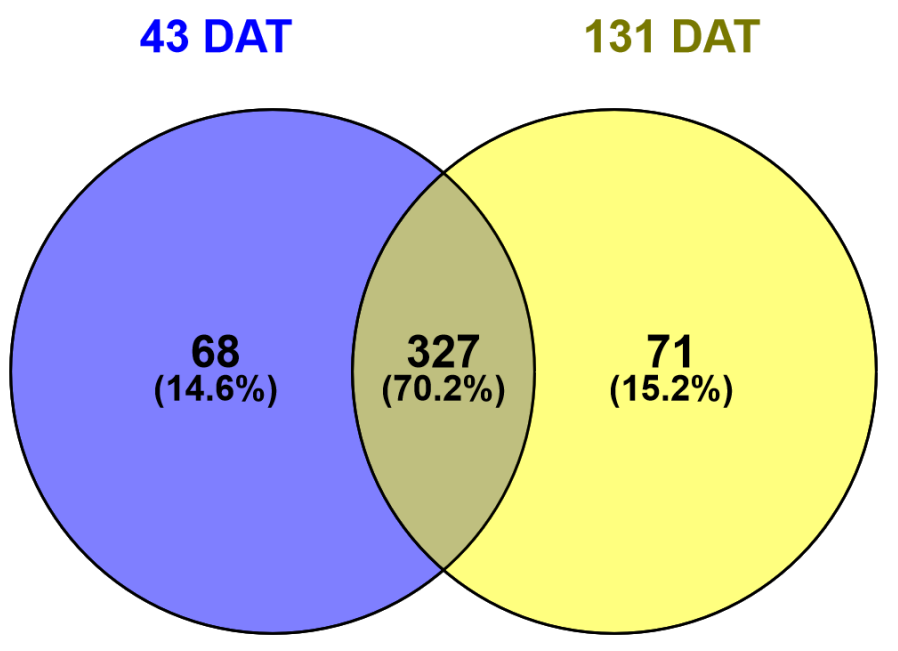

Figure 2. Venn diagram of statistically different metabolites $(\mathrm{P}<0.05)$ in pepper plants following treatment with microbial biostimulants. Compounds were profiled by untargeted metabolomics. Samples were harvested at two dates: 43 days after transplanting (DAT; vegetative stage), and 131 DAT (reproductive stage). 

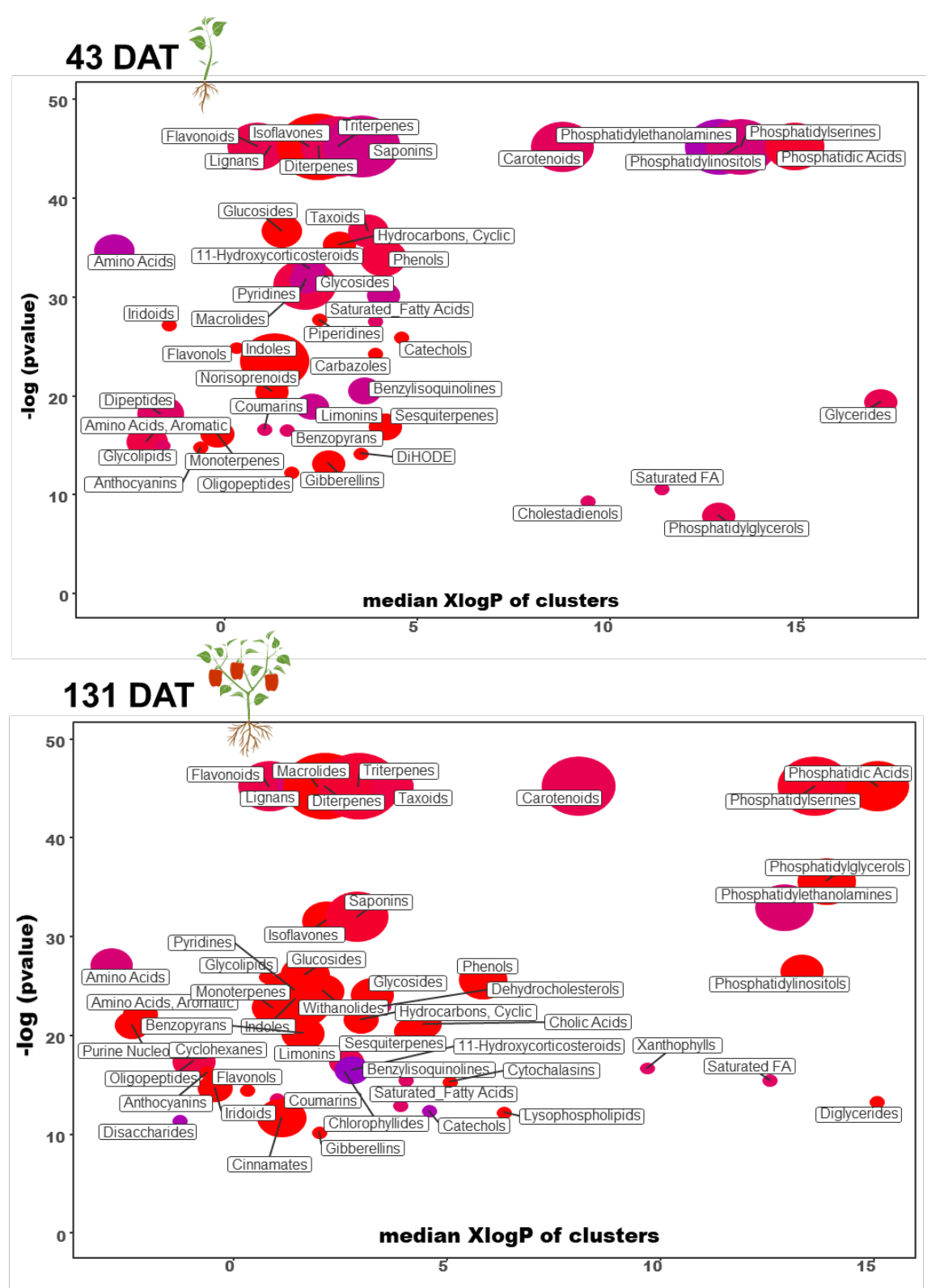

Figure 3. Chemical similarity enrichment analysis (ChemRICH) of statistically different annotated metabolites in microbial-based biostimulant treated leaves compared to untreated control at vegetative (43 days after transplanting; DAT) and reproductive stage (131 DAT). 

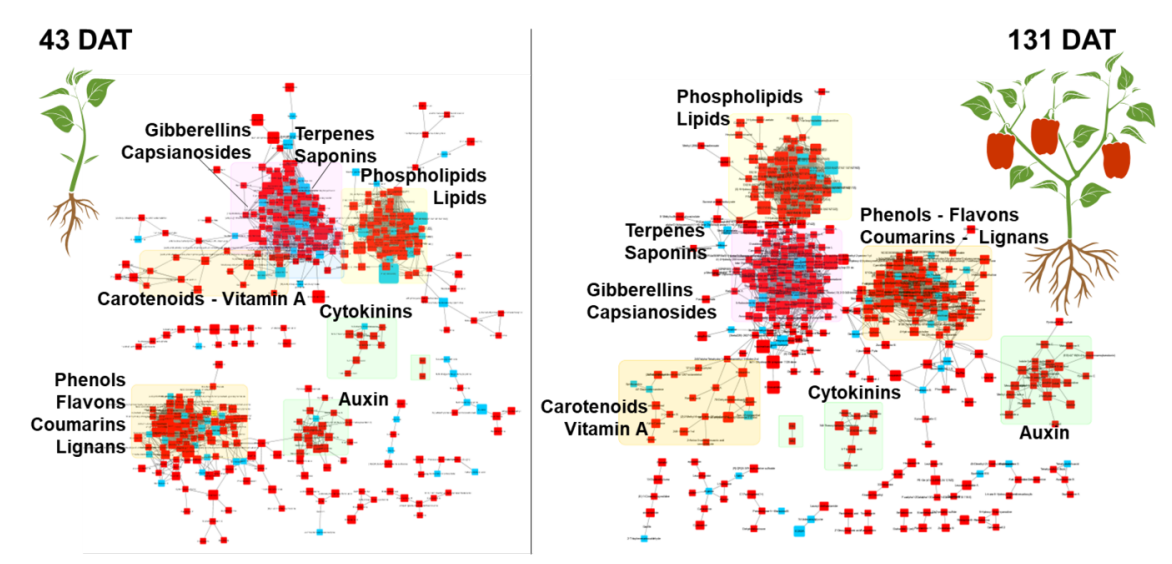

Figure 4. MetaMapp Metabolomic network maps of pepper leaves at vegetative (43 days after transplanting; DAT) and reproductive stage (131 DAT). Microbial-based biostimulant treated plants were compared to control ones. Red dots are compounds with an increase of fold change, while blue ones represent compounds with a decrease in fold changes. Chemical similarity and KEGG reaction were utilised to draw the clusters and nodes.

\section{Table 1}

Effect of microbial-based biostimulant application on fruit yield of greenhouse-grown peppers at different days after transplanting (DAT)

\begin{tabular}{llllll}
\hline Treatment & Fruit yield $\left(\mathrm{kg} \mathrm{plant}^{-1}\right)$ & Fruit yield $\left(\mathrm{kg} \mathrm{plant}^{-1}\right)$ & Fruit yield $\left(\mathrm{kg} \mathrm{plant}^{-1}\right)$ & ${\text { Fruit yield }\left(\mathrm{kg} \mathrm{plant}^{-1}\right)}$ Fruit \\
\hline & 139 DAT & 174 DAT & 243 DAT & 264 DAT & $272 \mathrm{D}$ \\
Control & 0.57 & 0.85 & 0.59 & 0.73 & 0.63 \\
Biostimulant & 0.73 & 1.41 & 0.48 & 0.74 & 0.81 \\
Significance & $*$ & $* *$ & $\mathrm{~ns}$ & $\mathrm{~ns}$ & $*$ \\
\hline
\end{tabular}

Two-tailed unpaired Student $t$ - test, ns $=$ nonsignificant, ${ }^{*} \mathrm{P}<0.05$, and ${ }^{*} \mathrm{P}<0.01$.

\section{Table 2}

Effect of microbial-based biostimulant application on fruit number of greenhouse-grown peppers at different days after transplanting (DAT)

\begin{tabular}{|c|c|c|c|c|}
\hline Treatment & Fruit number (n. plant ${ }^{-1}$ ) & Fruit number (n. plant ${ }^{-1}$ ) & Fruit number (n. plant $^{-1}$ ) & Fruit number (n. plant \\
\hline & 139 DAT & $174 \mathrm{DAT}$ & $243 \mathrm{DAT}$ & $264 \mathrm{DAT}$ \\
\hline Control & 2.35 & 3.65 & 2.90 & 2.20 \\
\hline Biostimulant & 2.75 & 5.56 & 1.92 & 1.98 \\
\hline Significance & ns & $* *$ & ns & ns \\
\hline
\end{tabular}

Two-tailed unpaired Student $t$ - test, ns $=$ nonsignificant, ${ }^{*} \mathrm{P}<0.05$, and ${ }^{*} \mathrm{P}<0.01$.

Table 3.

Effect of microbial-based biostimulant application on fruit mean weight of greenhouse-grown peppers at different days after transplanting (DAT) 


\begin{tabular}{|c|c|c|c|c|}
\hline Treatment & Fruit mean weight $\left(\mathrm{g} \mathrm{fruit}^{-1}\right)$ & Fruit mean weight $\left(\mathrm{g} \mathrm{fruit}^{-1}\right)$ & Fruit mean weight $\left(\mathrm{g}\right.$ fruit $\left.^{-1}\right)$ & Fruit mean w \\
\hline & $139 \mathrm{DAT}$ & $174 \mathrm{DAT}$ & 243 DAT & $264 \mathrm{DAT}$ \\
\hline Control & 244.0 & 232.4 & 204.4 & 335.1 \\
\hline Biostimulant & 264.7 & 254.4 & 250.4 & 368.3 \\
\hline Significance & $* *$ & $* *$ & ns & $*$ \\
\hline
\end{tabular}

Two-tailed unpaired Student $t$ - test, ns $=$ nonsignificant, ${ }^{*} \mathrm{P}<0.05$, and ${ }^{* *} \mathrm{P}<0.01$.

Table 4.

Effect of microbial-based biostimulant application on compound chemical classes (CHEMRICH) of greenhouse-grown peppers at vegetative stage (43 DAT)

\begin{tabular}{|c|c|c|c|c|c|}
\hline Cluster name & Cluster size & p-values & FDR & Key compound & In \\
\hline Carotenoids & 10 & $2.2 \mathrm{E}-20$ & $9.2 \mathrm{E}-20$ & Fucoxanthinol, Vit A, Alpha Carotene & 7 \\
\hline Diterpenes & 17 & $2.2 \mathrm{E}-20$ & $9.2 \mathrm{E}-20$ & NCGC00385284-01_C32H54O13 & 17 \\
\hline Flavonoids & 9 & $2.2 \mathrm{E}-20$ & $9.2 \mathrm{E}-20$ & Skullcapflavone I 2'-(2"-E-cinnamoylglucoside) & 7 \\
\hline Isoflavones & 6 & $2.2 \mathrm{E}-20$ & $9.2 \mathrm{E}-20$ & Genistein & 6 \\
\hline Lignans & 4 & $2.2 \mathrm{E}-20$ & $9.2 \mathrm{E}-20$ & Myricatomentoside I & 3 \\
\hline Phosphatidic Acids & 9 & $2.2 \mathrm{E}-20$ & $9.2 \mathrm{E}-20$ & $\mathrm{PA}(18: 0 / 18: 2)$ & 8 \\
\hline Phosphatidylethanolamines & 12 & $2.2 \mathrm{E}-20$ & $9.2 \mathrm{E}-20$ & $\mathrm{PE}(\mathrm{P}-16: 0 / 20: 5)$ & 4 \\
\hline Phosphatidylinositols & 5 & $2.2 \mathrm{E}-20$ & $9.2 \mathrm{E}-20$ & PIM4(18:1/14:0) & 5 \\
\hline Phosphatidylserines & 12 & $2.2 \mathrm{E}-20$ & $9.2 \mathrm{E}-20$ & $\mathrm{PS}(\mathrm{P}-16: 0 / 13: 0)$ & 7 \\
\hline Saponins & 15 & $2.2 \mathrm{E}-20$ & $9.2 \mathrm{E}-20$ & Borassoside A & 8 \\
\hline Triterpenes & 14 & $2.2 \mathrm{E}-20$ & $9.2 \mathrm{E}-20$ & Cussoracoside F & 10 \\
\hline Glucosides & 5 & $1.1 \mathrm{E}-16$ & $3.9 \mathrm{E}-16$ & Luteolin-4'-O-glucoside & 5 \\
\hline Amino Acids & 5 & $7.8 \mathrm{E}-16$ & $2.2 \mathrm{E}-15$ & Arginine & 2 \\
\hline Phenols & 6 & $1.7 \mathrm{E}-15$ & $4.5 \mathrm{E}-15$ & Gibbilimbol B & 5 \\
\hline Glycosides & 4 & 7.7E-14 & $1.7 \mathrm{E}-13$ & Melissoidesin D & 2 \\
\hline Macrolides & 10 & $2.6 \mathrm{E}-14$ & $5.9 \mathrm{E}-14$ & Capsianoside & 8 \\
\hline Piperidines & 3 & $9.1 \mathrm{E}-13$ & $1.9 \mathrm{E}-12$ & Andrachcinidine & 3 \\
\hline Iridoids & 3 & $1.5 \mathrm{E}-12$ & $3 \mathrm{E}-12$ & NCGC00168877-02_C15H20O8 & 3 \\
\hline Catechols & 3 & $5.4 \mathrm{E}-12$ & $9.9 \mathrm{E}-12$ & (S)-[8]-Gingerol & 3 \\
\hline Flavonols & 3 & $1.6 \mathrm{E}-11$ & $2.7 \mathrm{E}-11$ & Kaempferol & 3 \\
\hline Auxins & 12 & $6 \mathrm{E}-11$ & $9.8 \mathrm{E}-11$ & Indole-3-acetamide & 12 \\
\hline Glycerides & 4 & 3.7E-09 & $5.4 \mathrm{E}-09$ & DG(19:1(9Z)/22:4(7Z,10Z,13Z,16Z)/0:0)[iso2] & 3 \\
\hline Limonins & 4 & $5.9 \mathrm{E}-09$ & $8.4 \mathrm{E}-09$ & 11beta-Acetoxydihydrocedrelone & 2 \\
\hline Dipeptides & 6 & $1.2 \mathrm{E}-08$ & $1.7 \mathrm{E}-08$ & Ala-Phe & 4 \\
\hline Sesquiterpenes & 4 & $4.5 \mathrm{E}-08$ & $6.1 \mathrm{E}-08$ & Leucascandrolide A & 4 \\
\hline Coumarins & 3 & $5.8 \mathrm{E}-08$ & 7.6E-08 & Coumarin & 2 \\
\hline Monoterpenes & 4 & $9.6 \mathrm{E}-08$ & $1.2 \mathrm{E}-07$ & NCGC00384740-01_C21H34O9 & 4 \\
\hline Amino Acids, Aromatic & 5 & 0.0000002 & $2.5 \mathrm{E}-07$ & Tryptophan & 4 \\
\hline Glycolipids & 3 & $3.1 \mathrm{E}-07$ & $3.6 \mathrm{E}-07$ & Lyciumoside IV & 2 \\
\hline Anthocyanins & 3 & $3.7 \mathrm{E}-07$ & $4.3 \mathrm{E}-07$ & Cyanidine-3-O-sambubioside & 3 \\
\hline DiHODE & 3 & $6.8 \mathrm{E}-07$ & $7.6 \mathrm{E}-07$ & 8(R)-Hydroperoxylinoleic acid & 3 \\
\hline Gibberellins & 4 & 0.0000019 & 0.0000021 & Gibberellin A20 & 4 \\
\hline Oligopeptides & 3 & 0.0000047 & 0.000005 & Indole-3-acetyl-L-isoleucine & 3 \\
\hline Saturated FA & 3 & 0.000024 & 0.000025 & Capric acid & 2 \\
\hline Phosphatidylglycerols & 4 & 0.00037 & 0.00037 & PG(18:2/13:0) & 3 \\
\hline
\end{tabular}




\section{Table 5.}

Effect of microbial-based biostimulant application on compound chemical classes (CHEMRICH) of greenhouse-grown peppers at reproductive stage (131 DAT)

\begin{tabular}{|c|c|c|c|c|}
\hline Cluster name & Cluster size & p-values & FDR & Key compound \\
\hline Carotenoids & 12 & $2.2 \mathrm{E}-20$ & $1.3 \mathrm{E}-19$ & Fucoxanthinol, Vit A, Alpha Carotene \\
\hline Diterpenes & 15 & $2.2 \mathrm{E}-20$ & $1.3 \mathrm{E}-19$ & Traversianal \\
\hline Flavonoids & 9 & $2.2 \mathrm{E}-20$ & $1.3 \mathrm{E}-19$ & Skullcapflavone I 2'-(2"-E-cinnamoylglucoside) \\
\hline Lignans & 5 & $2.2 \mathrm{E}-20$ & $1.3 \mathrm{E}-19$ & Myricatomentoside I \\
\hline Macrolides & 9 & $2.2 \mathrm{E}-20$ & $1.3 \mathrm{E}-19$ & Capsianoside \\
\hline Phosphatidic Acids & 9 & $2.2 \mathrm{E}-20$ & $1.3 \mathrm{E}-19$ & PA(O-18:020:3(8Z11Z14Z)) \\
\hline Phosphatidylserines & 12 & $2.2 \mathrm{E}-20$ & $1.3 \mathrm{E}-19$ & $\mathrm{PS}(\mathrm{P}-16: 013: 0)$ \\
\hline Triterpenes & 15 & $2.2 \mathrm{E}-20$ & $1.3 \mathrm{E}-19$ & Tricalysioside $\mathrm{T}$ \\
\hline Phosphatidylglycerols & 8 & $3.3 \mathrm{E}-16$ & $1.7 \mathrm{E}-15$ & PG(P-18:017:2(9Z12Z)) \\
\hline Phosphatidylethanolamines & 8 & $4.9 \mathrm{E}-15$ & $2.1 \mathrm{E}-14$ & PE(P-16:020:5(5Z8Z11Z14Z17Z)) \\
\hline Saponins & 9 & $1.2 \mathrm{E}-14$ & $4.9 \mathrm{E}-14$ & Namonin E \\
\hline Isoflavones & 6 & $1.8 \mathrm{E}-14$ & $6.8 \mathrm{E}-14$ & Genistein \\
\hline Amino Acids & 5 & $1.6 \mathrm{E}-12$ & $5.4 \mathrm{E}-12$ & L-Valine \\
\hline Phosphatidylinositols & 5 & $3.1 \mathrm{E}-12$ & $1 \mathrm{E}-11$ & PIM4(18:1(9Z)14:0) \\
\hline Glucosides & 6 & $4.3 \mathrm{E}-12$ & $1.3 \mathrm{E}-11$ & Daedaleaside D \\
\hline Glycolipids & 3 & $5.3 \mathrm{E}-12$ & $1.5 \mathrm{E}-11$ & Capsoside A \\
\hline Phenols & 6 & $7 \mathrm{E}-12$ & $1.9 \mathrm{E}-11$ & Gibbilimbol B \\
\hline Glycosides & 5 & $3.2 \mathrm{E}-11$ & $7.6 \mathrm{E}-11$ & Cyclopassifloside VII \\
\hline Auxins & 12 & $5.1 \mathrm{E}-11$ & $1.2 \mathrm{E}-10$ & INDOLE-3-PYRUVIC ACID \\
\hline Monoterpenes & 5 & $1.2 \mathrm{E}-10$ & $2.5 \mathrm{E}-10$ & beta-Thujaplicin \\
\hline Amino Acids, Aromatic & 4 & $2.3 \mathrm{E}-10$ & $4.6 \mathrm{E}-10$ & 34-Dihydroxy-L-phenylalanine \\
\hline Purine Nucleosides & 4 & $6.9 \mathrm{E}-10$ & $1.2 \mathrm{E}-09$ & Adenosine \\
\hline Sesquiterpenes & 4 & $1.4 \mathrm{E}-09$ & $2.4 \mathrm{E}-09$ & $(+)$-vulgraon B \\
\hline Oligopeptides & 5 & $2.7 \mathrm{E}-08$ & $4.3 \mathrm{E}-08$ & Indole-3-acetyl-L-isoleucine \\
\hline Limonins & 4 & $2.9 \mathrm{E}-08$ & $4.4 \mathrm{E}-08$ & Toonaciliatin D \\
\hline Xanthophylls & 3 & $5.6 \mathrm{E}-08$ & $8.3 \mathrm{E}-08$ & Spirilloxanthin \\
\hline Chlorophyllides & 3 & $8.2 \mathrm{E}-08$ & $1.2 \mathrm{E}-07$ & chlorophyllide a \\
\hline Anthocyanins & 3 & $8.4 \mathrm{E}-08$ & $1.2 \mathrm{E}-07$ & Delphinidin-3-O-sambubioside \\
\hline Saturated FA & 3 & $1.8 \mathrm{E}-07$ & $2.5 \mathrm{E}-07$ & Petroformyne 1 \\
\hline Iridoids & 4 & $4.1 \mathrm{E}-07$ & $5.1 \mathrm{E}-07$ & Eleganoside B \\
\hline Flavonols & 3 & $5 \mathrm{E}-07$ & $6.1 \mathrm{E}-07$ & Kaempferol \\
\hline Coumarins & 3 & $1.3 \mathrm{E}-06$ & $1.5 \mathrm{E}-06$ & Coumarin \\
\hline Diglycerides & 3 & $1.7 \mathrm{E}-06$ & 0.000002 & DG(15:1(9Z)22:6(4Z7Z10Z13Z16Z19Z)0:0)iso2 \\
\hline Saturated_Fatty Acids & 3 & $2.5 \mathrm{E}-06$ & $2.8 \mathrm{E}-06$ & Capric acid \\
\hline Catechols & 3 & $4.3 \mathrm{E}-06$ & $4.8 \mathrm{E}-06$ & 33'44'-Tetrahydroxy-55'-diisopropyl-22'-dimethylbiph \\
\hline Lysophospholipids & 3 & $4.9 \mathrm{E}-06$ & $5.2 \mathrm{E}-06$ & $\mathrm{PC}(\mathrm{O}-17: 00: 0)$ \\
\hline Cinnamates & 6 & $8.2 \mathrm{E}-06$ & $8.5 \mathrm{E}-06$ & Sinapine \\
\hline Disaccharides & 3 & 0.000011 & 0.000011 & Melibiose \\
\hline Gibberellins & 3 & 0.000036 & 0.000036 & Gibberellin A20 \\
\hline
\end{tabular}

